Hyperreagible Thrombozyten

\title{
Nach Herzattacken benötigen Diabetiker eine stärkere Plättchenhemmung
}

— Die Thrombozyten von Diabetikern aggregieren leichter als diejenigen von Nichtdiabetikern. Deshalb sprechen Diabetiker nach einem akuten Koronarsyndrom (ACS) schlechter auf eine duale Standardtherapie mit ASS und Clopidogrel an, wie die CUREStudie zeigt: Das Risiko für klinische Komplikationen unter dualer Plättchenhemmung betrug bei Nichtdiabetikern 7,9\%, bei Diabetikern hingegen 14,2\%.

Der Grund: Bei Nichtdiabetikern versagt Clopidogrel bei $8 \%$ der Patienten, bei Diabetikern hingegen bei 36\%. Deshalb sollten Diabetiker nach ACS potentere Thrombozytenhemmer erhalten, erklärte Prof. Dominick J. Angiolillo, University of Florida in Jacksonville.

Dazu gibt es drei mögliche Partner für ASS: Clopidogrel in höherer Dosis, Ticagrelor, oder Prasugrel. Nur Prasugrel hat sich gegenüber der Clopidogrel-Standarddosierung als signifikant überlegen erwiesen: Bei den Diabetikern in der TRITONTIMI-38-Studie reduzierte Prasugrel gegen- über Clopidogrel die Ereignisrate (kardiovaskulärer Tod, Herzinfarkt, Schlaganfall) von $17 \%$ auf $12,2 \%$ (HR: 0,7). Gleichzeitig erwies sich die Blutungsrate (major bleedings bei Patienten ohne Bypasschirurgie) bei Diabetikern mit $2,6 \%$ vs. $2,5 \%$ als nicht erhöht.

Die Überlegenheit von Prasugrel gegenüber Clopidogrel nimmt mit der Dauer und Schwere der Diabeteserkrankung zu, ergänzte Angiolillo: Bei Patienten ohne Diabetes sank die Ereignisrate durch Prasugrel vs. Clopidogrel von $10,6 \%$ auf $9,2 \%$, bei insulinpflichtigen Diabetikern von $22,2 \%$ auf $14,3 \%$. Die relativen Risikoreduktionen gegenüber Clopidogrel waren jeweils signifikant und betrugen $14 \%$ bzw. $37 \%$.

\section{- Dirk Einecke}

Quelle: Jahrestagung 2011 der American Heart Association, Orlando, November 2011; Symposium „Acute Coronary Syndrome: Optimizing Outcomes Across the Spectrum of Care (Veranstalter: Daiichi-Sankyo, Lilly)

\section{Transmuraler Infarkt}

Prasugrel ist Clopidogrel überlegen

Bei transmuralem Herzinfarkt verhindert Prasugrel (Efient@) wirksamer als Clopidogrel weitere kardiovaskuläre Komplikationen, so das Ergebnis der 2007 publizierten TRITON-TIMI 38-Studie mit 13600 Patienten. Nun zeigt eine aktuelle, beim US-Herzkongress vorgestellte Subgruppenanalyse: Prasugrel ist sowohl bei primärer als auch bei verzögerter Koronarintervention überlegen. Der Vorteil war bei sekundärer $\mathrm{PCI}$ mit einer relativen Risikosenkung von 35\% und einer absoluten Risikosenkung von $4,6 \%$ besonders deutlich, ohne dass das Blutungsrisiko anstieg $(0,9 \%$ vs. $2,5 \%)$.

- Quelle: Jahrestagung 2011 der American Heart Association, Orlando, November 2011; Abstract 9309, http://circ.ahajournals.org/content/vol124/21_MeetingAbstracts;

\section{ACE-Hemmer/Kalziumantagonist in Fixkombination Bewährter Schutzschirm für Hypertoniker}

- Bei vielen Hypertonikern ist es mit Blutdrucksenkung allein meist nicht getan. Viele Patienten haben Stoffwechsel- oder Nierenprobleme, die ein effektives Risikomanagement erfordern. Im Behandlungsalltag hat sich dafür eine Fixkombination von ACE-Hemmer und Kalziumantagonist bewährt.

Der Einsatz von Fixkombinationen in der Hochdrucktherapie wirkt sich positiv auf die Therapietreue aus. Wie entscheidend das für den Behandlungserfolg sein kann, unterstreicht eine aktuelle Auswertung der ONTARGET-Studiendaten: NonAdhärenz hatte bei den kardiovaskulären Risikopatienten einen raschen und signifikanten Anstieg aller kardio- und zerebrovaskulären Endpunkte zur Folge.

\section{Mehr Schutz vor Herzinfarkt \& Co.} In der ACCOMPLISH-Studie z. B. nahm die Blutdruckkontrolle durch den Einsatz von Fixkombinationen deutlich zu. ACE-Hemmer plus Kalziumantagonist schützten bei gleicher Blutdrucksenkung hier effektiver vor Myokardinfarkt, Schlaganfall und Tod als ACE-Hemmer plus Diuretikum.

Aktuelle Erfahrungen aus dem Behandlungsalltag liefert eine nicht-interventionelle prospektive Multicenterstudie mit 622 meist vorbehandelten Hypertonikern (Scholze J et al. Expert Opin Pharmacother 2011, early online). Unter einer Fixkombination mit Enalapril und Lercanidipin (Zanipress ${ }^{\circledR}$ ) ging der mittlere Blutdruck innerhalb von drei Monaten von 164/95 mmHg auf 135/81 mmHg zurück (-29/-14 mmHg).
Der Anteil der Patienten mit mittelschwerer oder schwerer Hypertonie sank von $74,5 \%$ auf 7,4\%. 24-Stunden-Blutdruckmessungen zeigten eine gleichmäßige Senkung des Tages- und Nachtblutdrucks. Die prognostisch ungünstige Blutdruckvariabilität ging signifikant zurück, ebenso der Pulsdruck und die Mikroalbuminurie-Rate.

Lercanidipin macht weniger Ödeme als andere Kalziumantagonisten und hat antiproteinurische Effekte. Laut Prof. L.-C. Rump, Düsseldorf, konnte das auch bei Hypertonikern mit Proteinurie ( $>500$ $\mathrm{mg} / \mathrm{d}$ ), die mit einem RAS-Blocker vorbehandelt waren, gezeigt werden.

- Ulrike Wepner

5. Kardiovaskuläres Merckle-Recordati-Symposium, November 2011; Merckle Recordati 